\title{
Semi-Leptonic Decays of the F-Mesons and the Ensuing Lepton Spectrum.
}

\author{
A. Ali $\left(^{*}\right)$ \\ II. Institut fūr Theoretische Physik der Universität - Hamburg \\ T. C. YANG $\left(^{* *}\right)$ \\ Deutsches Elektronen-Synchrotron DES $Y$ - Hamburg
}

(ricevuto il 12 Dicembre 1977)

Preliminary evidence for the charmed meson $F^{ \pm}$, through its nonleptonic decay mode $\eta \pi^{ \pm}$, has recently been reported by the DASP collaboration at DESY $\left({ }^{1}\right)$. The mass of the $\mathrm{F}^{ \pm}$constructed through the $\eta \pi^{ \pm}$-mode has a value $(2.03 \pm 0.06) \mathrm{GeV}$, with the mass of the $\mathrm{F}^{*}$ set at $(2.14 \pm 0.06) \mathrm{GeV}$. This establishes the $\mathrm{FF}$ and $\mathrm{FF}^{*}$ thresholds near 4.1 and $4.2 \mathrm{GeV}$, respectively. The same group has also presented inclusive electron spectra between $3.99 \mathrm{GeV}$ and $5.2 \mathrm{GeV}$ c.m. energy ( $\left.{ }^{2}\right)$. The events, characterized by a single electron plus at least two charged tracks, are divided into three energy regions, namely, i) $3.99<E_{\text {c.m. }}<4.08 \mathrm{GeV}$, ii) $4.08<E_{\text {c.m. }}<4.52 \mathrm{GeV}$ and iii) $4.52<E_{\text {c.m. }}<5.2 \mathrm{GeV}$. The division is made with the view of studying the semi-leptonic decay spectrum coming from i) the D-decays alone, ii) the possible effects due to the F-production and decay, and iii) the effects due to possible charmed-baryon production and decays. The qualitative features of the electron spectra in the three energy regions are not very different, but a more detailed analysis is probably necessary in order to notice any difference. An immediate theoretical question is: How does the lepton spectrum from the $\mathbf{F}^{ \pm}$semi-leptonic decays compare with the one from the D-decays?

We give in this note a quantitative estimate of the semi-leptonic decay modes of the F-meson. The semi-leptonic decays of the D-mesons were previously studied by the present authors $\left({ }^{3}\right)$ and by others $\left({ }^{4}\right)$. The qualitative features of the electron spec-

\footnotetext{
(*) Work supported in part by the Bundesministerium für Forschung und Technologie.

(**) Address after September 1, 1977: Department of Physics and Astronomy, University of Massachusetts, Amherst, Mass., USA.

(1) DASP Collaboration, R. BRaNDelik et al.: DESY 77/44 (1977).

(2) W. Braunschweig, H.-U. Martyn, H. G. Sander, D. Schmitz, W. Stcrm, W. Wallraff,

D. Cords, R. Felst, R. Fries, E. Gadermann, B. Gittelman, H. Hultschig, P. Joos, W. Koch,

U. Kötz, H. Krehbiel, D. Kreinick, W. A. McNeely, K. C. Moffeit, P. Petersen, O. Römer,

R. Rüsch, B. H. Wilk, G. Wolf, G. Grindhammer, J. Ludwig, K. H. Mess, G. Poklz, J. Ringel, K. Saubrgerg, P. Schmüser, W. De Boer, G. Bugchhorn, B. Gunderson, R. Kotthaus, h. Lierl, H. Oberlack, M. Schliwa, S. Orito, T. SUDA, Y. Totsuka and S. Yamada: Phys. Lett., 63 B, 471 (1976);

R. BRandelik et al.: DESY Report (July 1977).

$\left({ }^{8}\right)$ A. ALI and T. C. YANG: Phys. Lett., 65 B, 275 (1976).

(4) A. AxI and Z. Z. AYDIN: DESY Report (in press); I. HINGLrFFe and C. H. LLEWELLFN-SMITH: Nucl. Phys., 414 B, 45 (1976); F. Bletzacker, H. T. NieH atd A. SoNi: Stony Brook preprint
} 
trum in the interval $3.99<E_{\text {c.m. }}<4.08 \mathrm{GeV}$ are well described by the decay modes $\mathrm{D} \rightarrow \mathrm{Ke} \bar{v}_{e}$ and $\mathrm{D} \rightarrow \mathrm{K}^{*} \mathrm{e}_{\mathrm{e}}$. This means presumably that the multi-hadronic decay modes do not contribute significantly to the semi-leptonic decays. We shall adopt a similar attitude toward the semi-leptonic decays of the $\mathrm{F}^{ \pm}$meson, namely, it is an equally good approximation to neglect the multi-hadron semi-leptonic decay modes of the F-meson. The semi-leptonic decays of the F-meson are different from that of the $D$-mesons in the following respect : there are more low lying single-particle modes available to the $\mathrm{F}^{ \pm}$-meson than to the $\mathrm{D}$-mesons. Thus, a priori, the lepton spectrum from F-decays could be different from that of the D-meson decays (in details).

We assume the standard $\Delta c=\Delta s$ rule. Since in the standard eharm model both the charm-changing charged weak current and the $F$-meson are isosinglets, the semileptonic decays of $\mathbf{F}$ involve only final states with $I=Y=0$. We consider only the following low lying single-particle decay modes:

$$
\left\{\begin{aligned}
F^{ \pm} & \rightarrow \eta^{ \pm} v_{v_{l}}, \\
& \rightarrow \eta^{\prime} \ell^{ \pm} v_{l}, \\
& \rightarrow \varphi^{ \pm \pm} v_{l}, \\
& \rightarrow \omega^{ \pm} v_{l} .
\end{aligned}\right.
$$

The last decay mode of (1), namely, $\omega^{\ell^{ \pm} \nu_{l}}$, is suppressed by the Zweig rule, since $\omega$ has very little strange quark content (ideal mixing). But for the pseudoscalars, $\eta$ and $\eta^{\prime}$, since they are not ideally mixed, the amount of mixing is quite important. This introduces an additional complication which is not present in the decays of D-mesons. To a good approximation, the $\eta$ and $\eta^{\prime}$ mesons are $S U_{3}$ octet and singlet respectively,

$$
\eta \sim \eta_{8}=\frac{1}{\sqrt{6}}(u \bar{u}+d \bar{d}-2 s \bar{s}) \quad \text { and } \quad \eta^{\prime} \sim \eta_{0}=\frac{1}{\sqrt{3}}(u \bar{u}+d \bar{d}+s \bar{s}) .
$$

Dropping the additional mixing angle (between $\eta_{8}$ and $\eta_{0}$ ), one finds that

$$
\frac{\Gamma\left(\mathrm{F}^{ \pm} \rightarrow \eta^{\ell \pm \nu_{l}}\right)}{\Gamma\left(\mathrm{F}^{ \pm} \rightarrow \eta^{\prime} \ell^{ \pm} \nu_{l}\right)} \simeq 2 \times \text { phase space, }
$$

where the factor 2 comes simply from counting the ss quark content of $\eta_{8}$ and $\eta_{0}$. However, the small mixing angle between $\eta_{8}$ and $\eta_{0}$ (around 11 degrees) turns out to make significant modification to (2). If we express $\eta$ and $\eta^{\prime}$ as

$$
\left\{\begin{array}{l}
\eta=\cos \theta \eta_{8}+\sin \theta \eta_{0}=\eta_{\sigma} \cos \alpha-\eta_{s} \sin \alpha, \\
\eta^{\prime}=-\sin \theta \eta_{8}+\cos \theta \eta_{0}=\eta_{\sigma} \sin \alpha+\eta_{s} \cos \alpha
\end{array}\right.
$$

(where $\eta_{\sigma} \equiv(1 / \sqrt{2})(u \bar{u}+d \bar{d})$ and $\eta_{s}=s \bar{s}$ ), we find that $\theta \simeq 11^{\circ}$ corresponds to $\alpha \simeq 43.7^{\circ}$, and thus the probability of finding s $\vec{s}$ pair in the $\eta$-meson is about the same as in $\eta^{\prime}$. Consequently we find

$$
\frac{\Gamma\left(\mathbf{F}^{ \pm} \rightarrow \eta^{ \pm} \boldsymbol{v}_{\ell}\right)}{\Gamma\left(\mathbf{F}^{ \pm} \rightarrow \eta^{\prime} \ell^{ \pm} \nu_{\ell}\right)} \simeq 0.92 \times \text { phase space. }
$$

(The number 0.92 becomes 1 if one uses $\alpha=45^{\circ}$ ).

ITP-SB-76-62; K. KaJANTte: Phys. Lelt., 65 B, $69(1976)$; B. Barger, T. GotTschalk and R. J. N. Phillips: Wisconsin preprint Coo-569; M. Gronau, C. H. Llewellyx-Smith, T. F. Walsh, S. WolFraM and T. C. YaNG: Nucl. Phys., 12 B, 47 (1977). 
In deriving (4), we have assumed $S U_{3}$ symmetry for the ratio of the form factors $f_{+}(0)$ of the above two processes. Since $S U_{3}$ symmetry-breaking correction to the difference of the form factors at zero momentum transfer comes in second order $\left(^{*}\right)$, we have reasons to believe that (4) provides a good approximation (up to the uncertainty of the mixing angle). (4) could be checked experimentally.

What remains to be calculated now is the branching ratio of $\mathrm{F} \rightarrow \eta l_{\nu}$ and $\mathrm{F} \rightarrow \varphi l v$. We define as in ref. $\left({ }^{3}\right)$ the following form factors:

$$
\left\langle\eta(k)\left|V_{\mu}^{\Delta c=\Delta s}\right| F(p)\right\rangle=f_{+}\left(q^{2}\right)(p+k)_{\mu}+f_{-}\left(q^{2}\right) q_{\mu},
$$

where $q=p-k$. Here only the vector current contributes. For $\mathrm{F} \rightarrow$ plv we have the form factors of the vector and axial-vector currents as given by

$$
\left\{\begin{array}{l}
\left\langle\varphi(k)\left|V_{\mu}\right| F(p)\right\rangle=i F_{1}^{\mathbf{V}}\left(q^{2}\right) \varepsilon_{\mu v \lambda \sigma} \varepsilon^{\nu} k^{\lambda} q^{\sigma}, \\
\left\langle\varphi(k)\left|A_{\mu}\right| F(p)\right\rangle=F_{1}^{\mathbf{A}}\left(q^{2}\right) \varepsilon_{\mu}+F_{2}^{\mathbf{A}}\left(q^{2}\right)(\varepsilon \cdot p) k_{\mu}+F_{3}^{\mathbf{A}}(\varepsilon \cdot p) q_{\mu},
\end{array}\right.
$$

where $\varepsilon$ is the polarization vector of the $\varphi$-meson.

As in ref. $\left({ }^{3}\right)$, we employ the current algebra hard meson technique $\left({ }^{5}\right)$ to calculate the various form factors. However, in the case of $F \rightarrow \eta l v$ (or $\eta^{\prime} l v$ ), the method is not useful, since we do not know the mixing among the hitherto poorly understood $I=Y=0$ axial-vector mesons. But since the form factors $f_{+}(0)$ for $\mathrm{F} \rightarrow \eta l_{\nu}$ and $\mathrm{D} \rightarrow \mathrm{K} \ell_{\nu}$ are related by $S U_{3}$, and as remarked above ( $\left.{ }^{*}\right)$ the $S U_{3}$ breakings to the difference of them appears in second order, we take the $f_{+}(0)$ of $\mathrm{F} \rightarrow \eta l_{\nu}$ as given by $S U_{3}$ symmetry. We assume for simplicity a monopole form factor $\left({ }^{* *}\right)$. One finds

$$
f_{+}^{\eta}\left(q^{2}\right)=f_{+}^{\eta}(0) \frac{m_{\mathrm{F}^{*}}^{3}}{m_{\mathrm{F}^{*}}^{2}-q^{2}}=\frac{1}{\sqrt{2}} \frac{m_{\mathrm{F}^{*}}^{2}}{m_{\mathrm{F}^{*}}^{2}-q^{2}} .
$$

The contribution of the $q_{\mu}$ term in (5), being proportional to $m_{l}$ in the decay rate, can be neglected.

For the decay of $\mathrm{F} \rightarrow p^{l v}$, the axial-vector form factors are given by current-algebra hard-meson technique as previously applied to the $\mathrm{D} \rightarrow \mathrm{K} * l_{\nu}$ decay, we find similarly

$$
\left\{\begin{array}{l}
\left.F_{1}^{A}\left(q^{2}\right)=\sqrt{2} m_{\varphi}\left[1+\frac{1}{2\left(q^{2}-m_{F_{A}}^{2}\right.}\right)\left\{\left(m_{\varphi}^{2}-q^{2}\right)+\frac{\delta}{2}\left(m_{\mathrm{F}}^{2}+m_{\varphi}^{2}-q^{2}\right)\right\}\right] \\
F_{2}^{A}\left(q^{2}\right)=-\frac{\delta m_{\varphi}}{\sqrt{2}\left(q^{2}-m_{\mathrm{F}_{\mathbf{A}}}^{2}\right)}
\end{array}\right.
$$

where $\delta$ is a parameter as in ref. $\left({ }^{3}\right)$. The above result (8) already takes into account the $S U_{3}$ symmetry between $\mathrm{D} \rightarrow \mathrm{K} * \ell_{\nu}$ and $\mathrm{F} \rightarrow \varphi$ lv. As in ref. $\left({ }^{3}\right)$, we have assumed $F_{\mathbf{D}}=F_{F}=F_{\mathbf{K}}=F_{\pi k}$, where the $F^{\prime}$ 's are the (axial-vector current) decay constants of

(*) This is a corollary of the Ademollo-Gatto theorem. Let $\lambda H^{\prime}$ be the $S U_{1}$ symmetry-breaking term. Under $S U_{3}$ transformation, $\lambda H^{\prime}=\lambda_{3} u_{3}+\lambda_{8} u_{3}+\lambda_{15} u_{15}$ with obvious notations. By Ademollo-Gatto theorem, the $S U_{*}$ corrections to $f_{+}(0)$ come in second order of $\lambda$. But the difference of the two $f_{+}(0)$ does not depend on $\lambda_{15}$, therefore the $S U_{s}$ correction to the difference of the two $f_{+}(0)$ comes in second order of $\lambda_{3}, \lambda_{3}$.

(5) H. J. Schnitzer and S. Weinberg: Phys. Rev., 464, 1828 (1967).

(**) This corresponds to $\delta=-1$ for the $D \rightarrow K e \bar{v}_{\mathrm{e}}$ form factor as given in ref. $\left({ }^{3}\right)$. 
the pseudoscalar mesons. The vector form factor of $\mathrm{F} \rightarrow \varphi l_{\nu}$ is not determined by the current algebra, but is related to $\mathrm{D} \rightarrow \mathrm{K} * l_{\nu}$ by $S U_{3}$. We have, in the case of $\mathrm{D} \rightarrow \mathrm{K} * \ell_{v}$, considered two approaches: 1) exact $S U_{4}$ relation for the three-point vertices, 2) broken $\$ U_{4}$ corrections via the Gell-Mann-Okubo ansatz $\left(^{6}\right)$. This gives for $F \rightarrow \varphi l v$, in pole-dominated form

$$
F_{1}^{\nabla}\left(q^{2}\right)=\frac{\sqrt{2} g_{\mathrm{F}^{*}} g_{\mathrm{F}^{*} \varphi \mathrm{F}}}{q^{2}-m_{\mathrm{F}^{*}}^{2}}=\frac{\sqrt{2}\left(\left(m_{\mathrm{F}^{*}} / m_{\rho}\right) g_{\rho}\right)\left(g_{\omega \rho \pi} / \sqrt{2}\right)}{q^{2}-m_{\mathrm{F}^{*}}^{2}}=\frac{m_{\mathrm{F}^{*}} m_{\rho}}{\sqrt{2}}\left[\frac{3 \Gamma(\omega \rightarrow \pi \gamma)}{\alpha k^{3}}\right]^{\frac{1}{2}},
$$

where we assumed exact $S U_{4}$ symmetry for $g_{F^{*} \varphi \mathrm{F}}$ and $g_{\omega \rho \pi} . g_{\mathrm{F}^{*}}$ is defined by $\left\langle 0\left|\nabla_{\mu}\right| F^{*}\right\rangle=g_{\mathrm{F}^{*}} \varepsilon_{\mu}$. If we take into consideration $S U_{4}$ symmetry breaking $\grave{a}$ la GellMann-Okubo, $g_{F^{*} \varphi F}$ decreases by about $30 \%$ compared with $(8)\left({ }^{3}\right)$. Since the vector form factor does not give the dominant contribution, the $S U_{4}$ symmetry-breaking corrections will not appreciably affect the decay rates.

TABLE I. - Decay rates and the relative rates for the semi-leptonic decays of the $\mathrm{F}^{ \pm}$-mesons with $m_{\mathrm{F}}=2.03 \mathrm{GeV}$ and $S U_{4}$ symmetry assumed for $F_{1}^{\mathrm{V}}$ (see test).

\begin{tabular}{|c|c|c|c|}
\hline \multirow[t]{2}{*}{ Mode } & \multicolumn{3}{|c|}{ Decay Widths } \\
\hline & \multicolumn{3}{|l|}{ Units $\left(s^{-1}\right)$} \\
\hline $\mathbf{F} \rightarrow \eta \theta \bar{v}_{\theta}$ & \multicolumn{3}{|l|}{$1.2 \cdot 10^{11}$} \\
\hline $\mathrm{F} \rightarrow \eta^{\prime} \mathrm{e}_{\mathrm{e}}$ & \multicolumn{3}{|l|}{$0.35 \cdot 10^{11}$} \\
\hline \multirow[t]{2}{*}{$\mathbf{F} \rightarrow \varphi \mathrm{e} \overline{\mathbf{v}}_{\mathrm{e}}$} & $\delta=0$ & $\delta=-0.5$ & $\delta=-1.0$ \\
\hline & $0.49 \cdot 10^{11}$ & $0.57 \cdot 10^{11}$ & $0.66 \cdot 10^{11}$ \\
\hline$\Gamma\left(\mathbf{F} \rightarrow \eta^{\prime} \mathrm{e}_{\mathrm{e}}\right) / \Gamma\left(\eta \bar{v}_{\mathrm{e}}\right)=$ & \multicolumn{3}{|l|}{0.29} \\
\hline \multirow[t]{2}{*}{$\Gamma\left(\mathbf{F} \rightarrow \varphi \mathrm{e} \overline{\mathrm{e}}_{\mathrm{e}}\right) / \Gamma\left(\mathrm{F} \rightarrow \eta \mathrm{e} \overline{\mathrm{e}}_{\mathrm{e}}\right)=$} & $\delta=0$ & $\delta=-0.5$ & $\delta=-1$ \\
\hline & 0.4 & 0.48 & 0.55 \\
\hline
\end{tabular}

TABLE II. - The same as for table $I$, with the $S U_{4}$ symmetry breaking for $F_{1}^{\mathbf{V}}$ calculated à la Gell-Mann-Okubo.

\begin{tabular}{llll}
\hline \multirow{2}{*}{ Mode } & \multicolumn{2}{l}{ Decay Widths } & \\
\cline { 2 - 4 } & Units $\left(\mathrm{s}^{-1}\right)$ & & \\
\hline $\mathrm{F} \rightarrow \eta \mathrm{e} \bar{v}_{\mathrm{e}}$ & $1.2 \cdot 10^{11}$ & & \\
\hline $\mathrm{F} \rightarrow \eta^{\prime} \mathrm{e} \bar{v}_{\mathrm{e}}$ & $0.35 \cdot 10^{11}$ & & $\delta=-1.0$ \\
\hline $\mathrm{F} \rightarrow \varphi \mathrm{e} \bar{v}_{\mathrm{e}}$ & $\delta=0$ & $\delta=-0.5$ & $0.63 \cdot 10^{11}$ \\
\cline { 2 - 4 } & $0.45 \cdot 10^{11}$ & $0.54 \cdot 10^{11}$ & \\
\hline$\Gamma\left(\mathrm{F} \rightarrow \eta^{\prime} \mathrm{e} \bar{v}_{\mathrm{e}}\right) / \Gamma\left(\mathrm{F} \rightarrow \eta \overline{\mathrm{v}}_{\mathrm{e}}\right)=$ & 0.29 & & $\delta=-1.0$ \\
\hline$\Gamma\left(\mathrm{F} \rightarrow \varphi \mathrm{e} \overline{\mathrm{e}}_{\mathrm{e}}\right) / \Gamma\left(\mathrm{F} \rightarrow \eta \mathrm{e} \bar{v}_{\mathrm{e}}\right)=$ & $\delta=0$ & $\delta=-0.5$ & 0.53 \\
\hline
\end{tabular}

(') A. KAZI, G. Kramer and D. H. Schiller: DESY $76^{5} 18$ (1976). 
Taking the $\mathrm{F}$ and $\mathrm{F}^{*}$ masses from ref. (1), i.e. $m_{\mathrm{F}}=2.03 \mathrm{GeV}, m_{\mathrm{F}^{*}}=2.14 \mathrm{GeV}$, our numerical results using eqs. (3)-(8) are given in table $I$ for $F_{1}^{\mathrm{V}}$ given by (9) and table II for $F_{1}^{\mathrm{V}}$ including the $S U_{4}$ symmetry breaking. We take $\delta$ to vary from 0

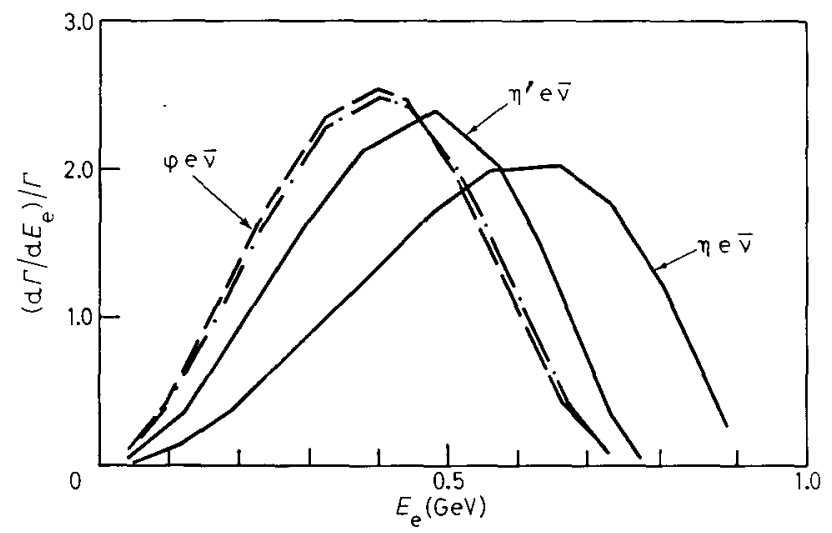

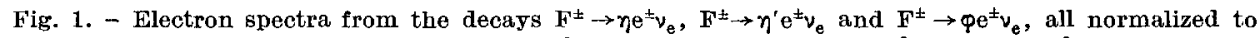

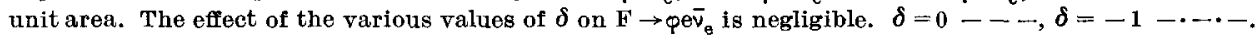

to -1 . The electron spectrum for each individual decay modes are shown in fig. 1 . Up to the $q^{2}$-dependence, the peaking of the electron energy distribution reflects essentially the kinematies of phase space. In fig. 2 we plot the inclusive electron spectrum after summing over the decay modes, with normalization as determined in table I.

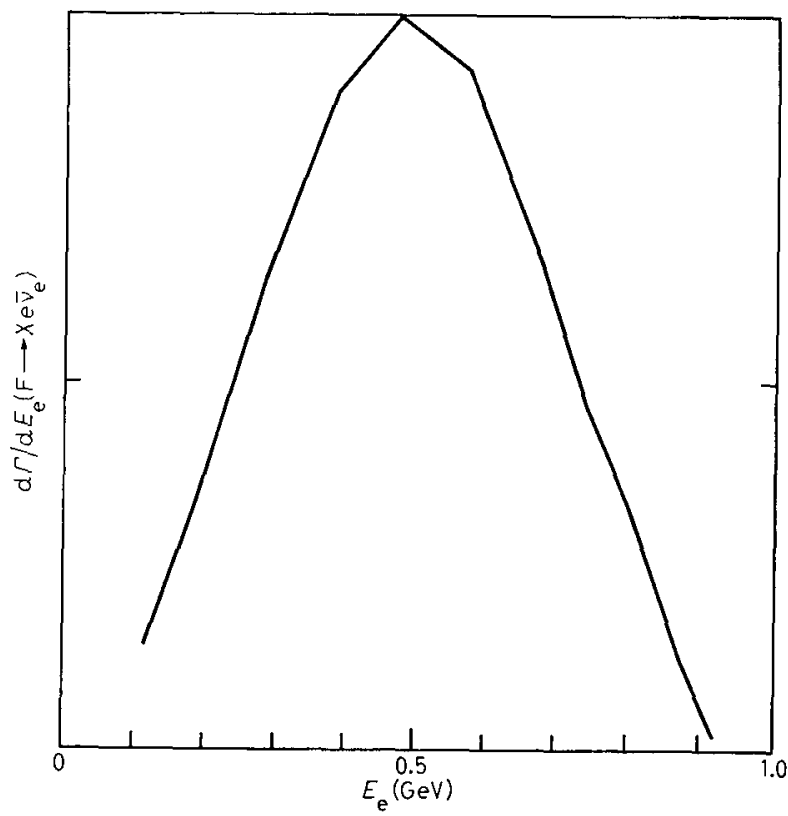

Fig. 2. - Inclusive electron spectrum for the semi-leptonic decays of the $F^{ \pm}$-mesons, normalized with $\Gamma\left(\mathbf{F} \rightarrow \eta^{\prime} \mathrm{e} \bar{v}_{\mathrm{e}}\right) / \Gamma\left(\mathrm{F} \rightarrow \eta \mathrm{e} \bar{v}_{\mathrm{e}}\right)=0.3, \Gamma\left(\mathbf{F} \rightarrow \varphi \mathrm{e} \bar{v}_{\mathrm{e}}\right) / \Gamma\left(\mathbf{F} \rightarrow \eta \overline{\mathrm{v}}_{\mathrm{e}}\right)=0.55$. 
Comparing the electron spectrum from the F-decay with that of the D-decays, we note that the decay modes $\mathrm{F} \rightarrow \eta_{\mathrm{e}} \bar{v}_{\mathrm{e}}$ and $\mathrm{F} \rightarrow \varphi \mathrm{e} \bar{v}_{\mathrm{e}}$ are very similar to $\mathrm{D} \rightarrow \mathrm{Ke} \overline{\mathrm{v}}_{\mathrm{e}}$ and $\mathrm{D} \rightarrow \mathrm{K}^{*} \mathrm{e} \bar{v}_{\mathrm{e}}$, respectively. The semi-leptonic decays of $\mathrm{F}$ have an additional decay mode $\mathbf{F} \rightarrow \eta^{\prime} \mathrm{e} \bar{v}_{\mathrm{e}}$, which tends to make the electron spectrum softer (by phase space). If $\eta^{\prime} \bar{e}_{\mathrm{e}}$ were an important mode of the semi-leptonic decays, the difference between the electron spectrum from F- and D-decays would be noticeable. Our calculation indicates that only $15 \%$ of the semi-leptonic F-decays goes into $\eta^{\prime} \bar{v}_{\mathrm{e}}$ and thus we do not anticipate any major change in the electron spectrum when the F-meson is produced in $e^{+} e^{-}$annihilation. The data $\left(^{2}\right)$ do not indicate any major change in the electron spectrum as $E_{\text {c.m. }}$ moves from 4 to $5 \mathrm{GeV}$.

To conclude, we note that the nonleptonic decays of the F-meson are very different from those of the $\mathrm{D}$-mesons. If we could use the signatures of the nonleptonic decays of the $F$ to separate the electron spectrum of the F-mesons from that of the D-mesons (e.g. the electron spectrum from inclusive $\mathrm{e} \eta+\ldots v s$. eK $+\ldots$ ), we could test the semileptonic decays of the $\mathrm{F}$ and $\mathrm{D}$ mesons in a more quantitative way.

We would like to thank our experimental and theoretical colleagues at DESY and the University of Hamburg for useful discussions, H. Joos, and G. KRAMER for reading the manuscript. 